\title{
Experimenting with Sensor Webs Using Earth Observing 1
}

\author{
Dan Mandl \\ Code 584 \\ NASA/GSFC \\ Greenbelt, Md. 20771 \\ $301-286-4323$ \\ Daniel.J.Mandl@nasa.gov
}

Abstract - The New Millennium Program (NMP) Earth Observing 1 (EO-1) satellite was launched November 21, 2000 as a one year technology validation mission. After an almost flawless first year of operations, EO-1 continued to operate in a test bed mode to validate additional technologies and concepts that will be applicable to future sensor webs. A sensor web is a group of sensors, whether space-based, ground-based or air plane-based which act in a collaborative autonomous manner to produce more value than would otherwise result from the individual observations.

Interestingly, it seems that the trend at this time is to link a set of heterogeneous satellites and instruments together in an "ad hoc" constellation for a limited period of time to get new science products. In our experiments, we used the MODIS instruments on Aqua and Terra to locate terrestrial events such as forest fires and then to trigger high-resolution images of targeted events with either the Advance Land Imager (ALI) or Hyperion on EO-1. To achieve this, we created a variety of software on the spacecraft and on the ground to coordinate the planning and triggering of these images. Furthermore, it became clear from the lessons learned that future sensor webs are going to need some key capabilities to enable progressive autonomy and sufficient reuse to make these constellation cost effective for science. This paper describes the experiments, the lessons learned and the implications for future sensor webs.

Keywords: sensor webs, satellite constellations, progressive mission autonomy, EO-1, Hyperion, ALI

\section{TABLE OF CONTENTS}

1. INTRODUCTION

2. BRIEF EO-1 MISSION OVERVIEW

3. END-TO-END MESSAGING CAPABILITIES

4. PROGRESSIVE MISSION AUTONOMY

5. PLUG AND PLAY

6. CONCLUSION

7. REFERENCES

8. AUTHOR BIO

9. ACKNOWLEDGEMENTS

\section{INTRODUCTION}

Sensor webs will greatly enhance future science because they will allow more efficient use of on-orbit assets to perform such activities such as catching timely observations of transient events. In the case of EO-1, a low earth observing satellite with a polar orbit, each spot on the earth can only be seen in the nadir direction once every 16 days. Therefore, in order to catch events such as forest fires in high resolution, either there would have to be 16 or more copies of EO-1 to assure that a timely observation can be made or a more intelligent approach can be used whereby a low resolution survey instrument such as MODIS, which sees all of the earth each day, can detect key events such as fire and then alert other assets such as EO-1 to come take a closer look.

Interestingly, in the recent California fires, the need for this intelligent use of on-orbit assets was demonstrated in late October and early November 2003. The forestry service needed quick imagery of burned areas in southern California in order to efficiently deploy their assets to prevent erosion and mudslides in burned areas, which could result from the upcoming rainy season. Figure 1 shows one of the EO-1 Advanced Land Imager (ALI) images used by the Forestry Service which had been triggered by MODIS. Note that if this paper is being viewed in black and white; the burn areas highlighted in red will not be visible. The top map embedded in figure 1 is the MODIS active fire map with the small square within the map representing the area subsequently imaged with the high-resolution ALI.

Figures 2 and 3 show how the Forestry Service makes use of

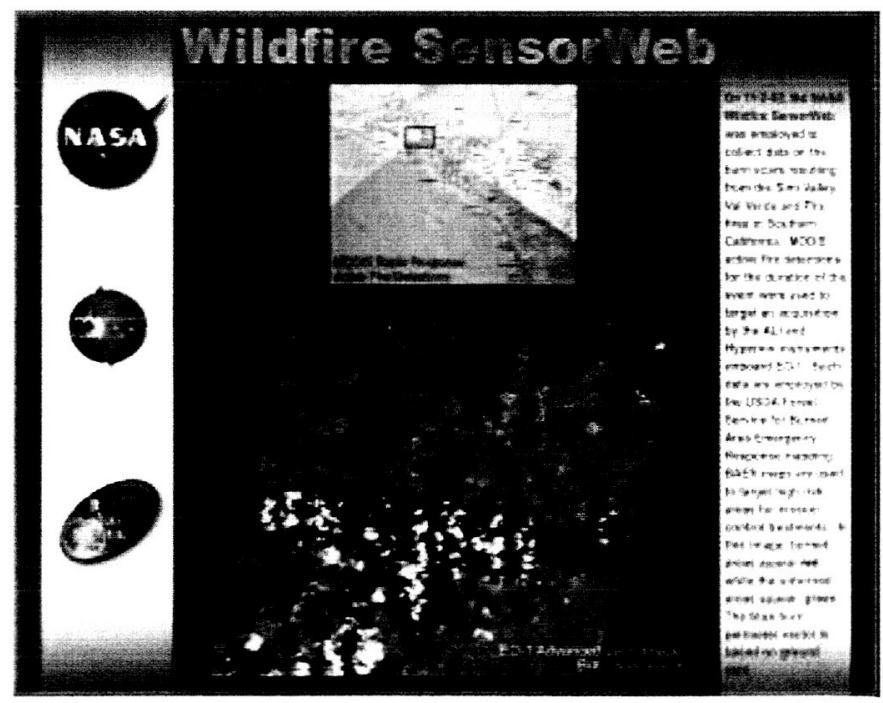

Figure 1 - EO-1 SensorWeb triggered ALI image of Simi Valley fire 11-2-03. Color-coding of fire only visible if this paper being viewed in color. 
this type of imagery. Figure 2 was a SensorWeb image of a fire in Glacier National Park in Montana on August 21, 2003. In particular, the Robert fire was being targeted. Note that in this figure, the Burned Area Reflectance Classification (BARC) map, which was created by the Burned Area Emergency Rehabilitation (BAER) team, is superimposed on top of the ALI image. If this paper is being viewed in color, one can note that the focus is on the red and yellow areas, thus allowing the team to concentrate treatment options on high-risk areas. The images are used to contract out work to avoid erosion and the BAER map allows classification into the areas more urgently needing work. Since the Forestry Service has limited resources and funding, it is essential that recovery assets be used efficiently. Figure 3 depicts members of a BAER team assessing fire damage.

Here are comments from Rob Sohlberg from Univ. of Maryland and Rob Sauer from the Forestry Service in response to the use of a diverse set of space assets including the EO-1 sensor web to gather the needed data:
Rob Sohlberg, University of Maryland:

"Many thanks to all who assisted in rapidly pulling together a comprehensive suite of post-fire imagery for the wildfires in Southern. Ca. Only by using the whole range of available airborne assets and spacecraft were we able to provide data for each incident in such a short time and with difficult weather conditions. In the end, each sensor has made a unique contribution."

\section{Rob Sauer, Forestry Service:}

"THANK YOU for all our efforts in providing imagery over the last two weeks! Considering our one L5 attempt within the window was cloudy and our "issues" with SPOT, the imagery from ALI, ASTER, MASTER, $M A S$, and MODIS proved invaluable. Several of the incidents only received imagery from these sensors and were actually quite pleased with the results".

The key aspect of this observation event that could portend things to come is the use of various space assets in ways

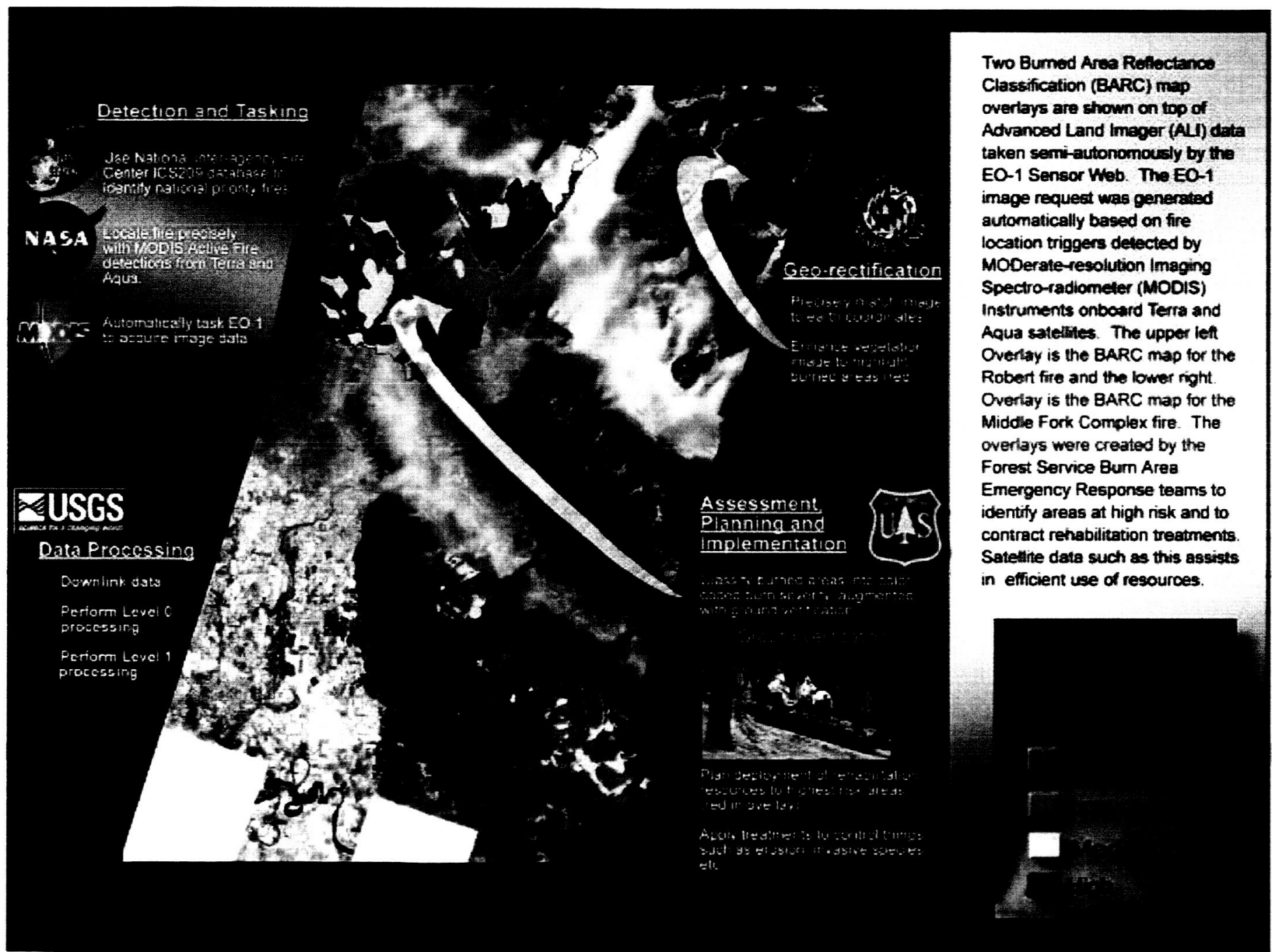

Figure 2 - EO-1 Sensor Web experiment of 8-21-03 in which MODIS "hot pixels" triggered EO-1 to take high resolution image. Image was converted into a Burn Area Reflectance Classification (BARC) map which was used by the Forestry Service Burn Area Emergency Rehabilitation team. 


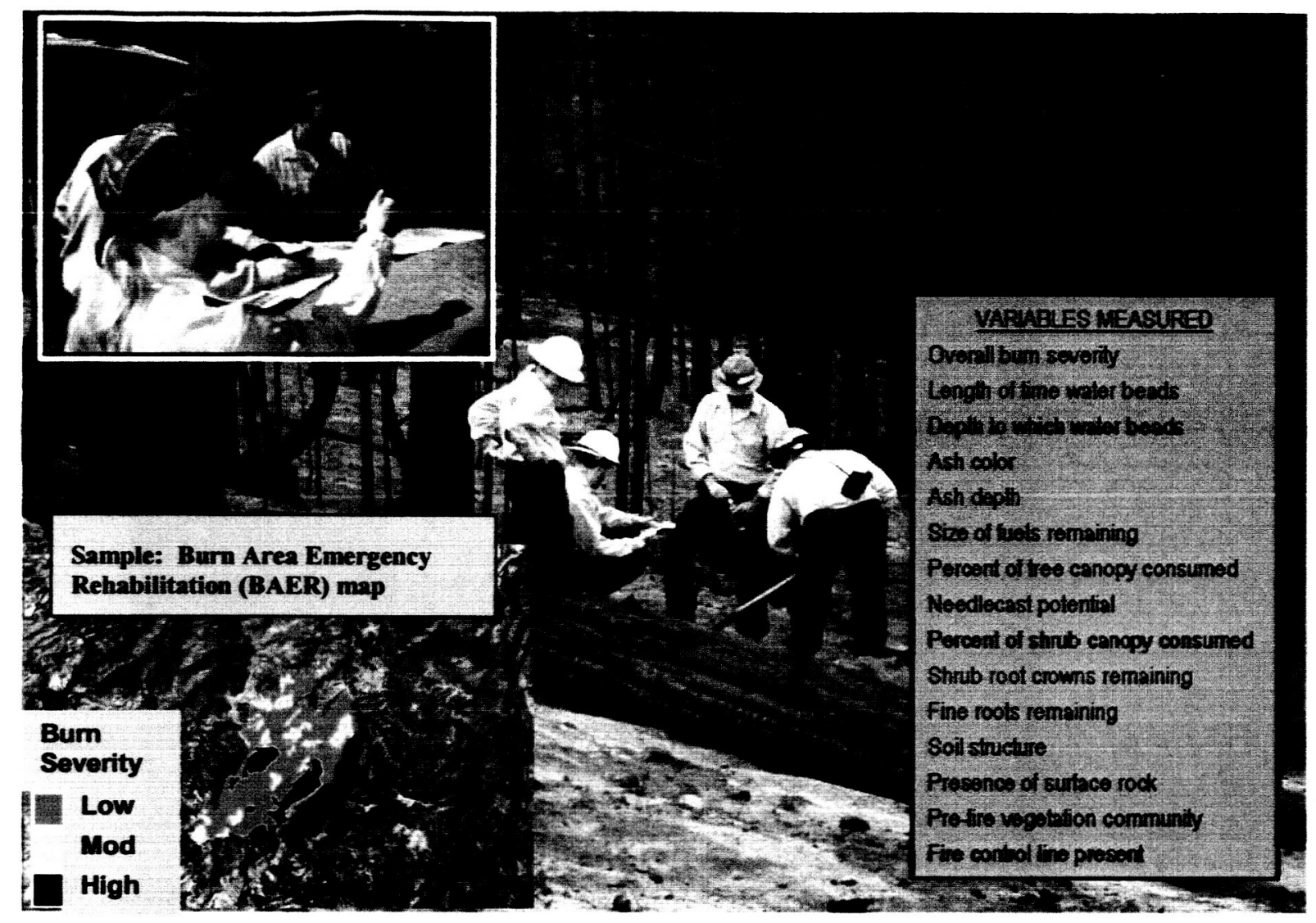

Figure 3 - Sample BAER map overlaid on picture of Forestry workers assessing fire damage.

other than that for which they were designed and the demonstrated capability to temporarily assemble an "ad hoc" constellation to increase the value of imagery collected in order to assess transient phenomena. There were and continue to be (as long as funding continues for EO-1) a variety of experiments centered around three key capabilities to enable sensor webs of the future, mission end-to-end messaging capability, progressive mission autonomy and plug and play. All three capabilities overlap somewhat and will be covered in sections 3,4 and 5 .

Section 2 provides a brief EO-1 mission overview and background. Section 5 provides some lessons earned and future directions. Section 6 is the conclusion.

\section{BRIEF EO-1 MISSION OVERVIEW}

The New Millennium Program's first Earth-observing mission (EO-1) is a technology validation mission. It is managed by the NASA Goddard Space Flight Center in Greenbelt, Maryland and launched in November 2000. EO1 flies in formation with three other satellites in what is termed the "Morning Constellation" as depicted in figure 4. The purpose of this mission was to flight-validate revolutionary technologies that will contribute to the reduction of cost and increase of capabilities for future land imaging missions. For EO-1's prime mission, there were five instrument, five spacecraft, and three supporting technologies to flight-validate during a year of operations. Following the first year of operations, the EO-1 mission entered its extended mission phase in which additional validations were performed which centered on the theme of enabling sensor webs.

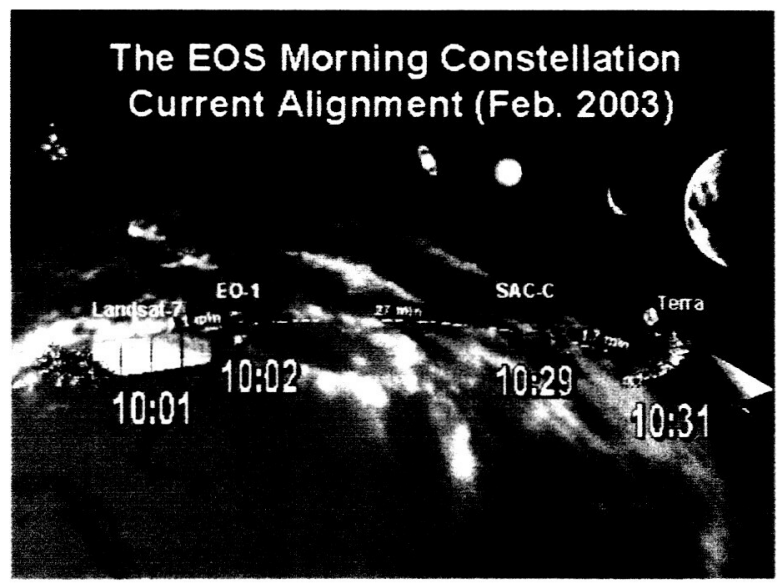

Figure 4 - EO-1 Mission Pictorial showing formation flying with Landsat, SAC-C and Terra 


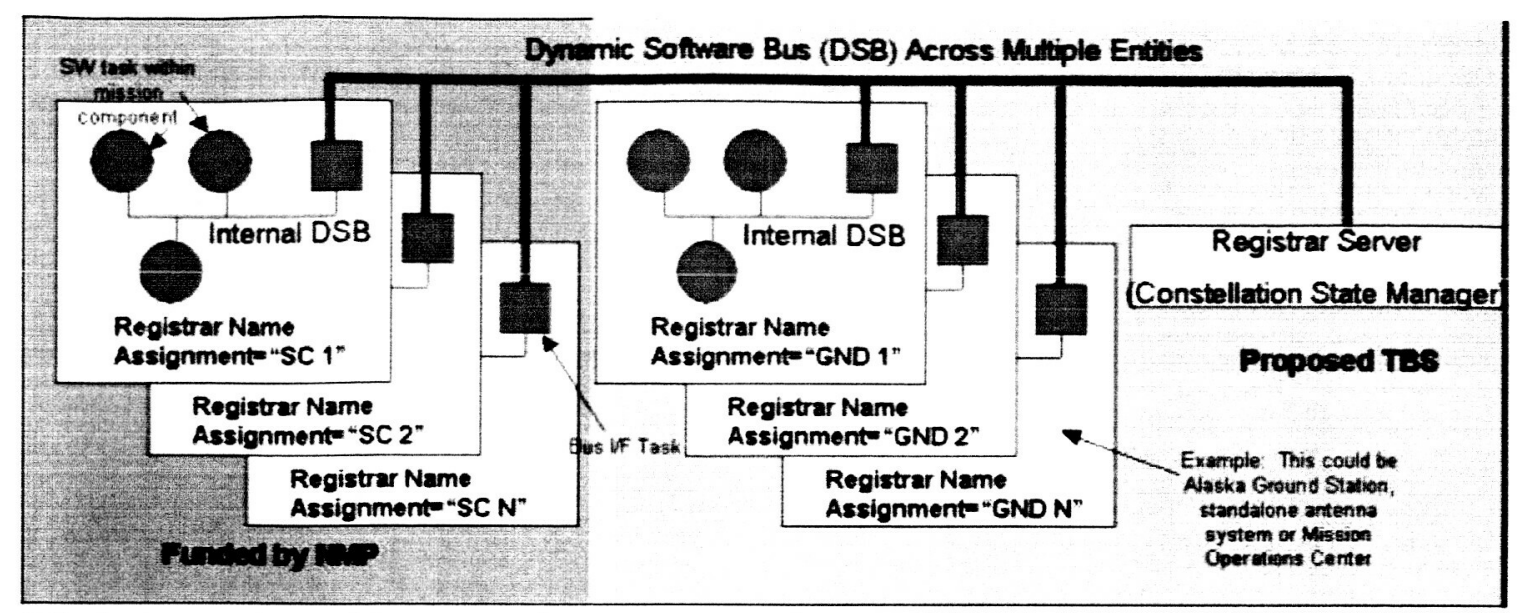

Figure 5 - Mission-messaging architecture so that messages can be passed from any process to any other process via naming convention similar to Internet

\section{Mission End-TO-End MesSaging}

\section{CAPABILITY}

The key to enabling the easy integration of heterogeneous components into a constellation is a messaging system whereby any software process can send a message to any other software process in the constellation merely by knowing the name, a dynamic software bus. Once this messaging system is in place then to set up progressive autonomy is more easily enabled since new processes can be more easily plugged in even while satellites are on-orbit. Instruments can easily coordinate via messages and scripts. In our case, we are demonstrating the onboard portion of the dynamic software bus via the use of Spacecraft Command Language (SCL) installed on EO-1 as a software backplane thus enabling other applications to plug in. In particular, we have begun to test CASPER onboard EO-1, which is an onboard planner. Figure 5 and 6 depict the architecture of this experiment. Figure 5 shows how SCL acts as the middleware to transform the existing software bus into a rudimentary dynamic software bus. The key difference between a software bus, which was built into EO-1, and a dynamic software bus is that both can send messages by name to any other process on the bus. However, the dynamic software bus can easily add new processes that can then be named and have messages sent to that process. This is a first step towards "plug and play" and would allow easier constellation resource management. Figure 7 shows conceptually how we integrated the SCL and CASPER into the existing flight SW architecture.

\section{Progressive Mission Autonomy}

For "ad hoc" constellations, the ability to easily link the assets, provide coordinated planning and augment the operations procedures whether flight or ground software needs to be added is essential. By adding both CASPER and SGM, we were able to conduct a whole host of experiments ranging from triggering via MODIS observations to cause an EO-1 observation, to our present endeavor which is to make some real time decisions using GOES active cloud cover detection to choose alternate scenes if one target is too cloudy. As the library of scripts increases, modifications of new operational scenarios have been getting easier. In Figure 8, see the operational scenario for the EO-1 Sensor Web forest. fires. There are similar scenarios for volcanic activity [2] and flood detection[3]. Figure 9 illustrates a conceptual diagram for implementation for a higher level of plug and play architecture for Sensor Webs. Figure 10 shows the funded tasks on the EO-1 testbed.

\section{Plug and Play}

Although the SCL/CASPER/SGM augmentation of EO-1 provided some degree of flexibility including a first step in "plug and play", it became clear that there were two key obstacles that would prevent easy development of future sensor webs. First, most projects are reticent to make changes on-orbit for fear that essential functionality would be inadvertently compromised. EO-1 has two Mongoose processors, each with $256 \mathrm{Mbytes}$ of memory. We therefore could isolate our onboard dynamic software bus into an essential and non-essential bus thus isolating new experiments from core functionality via a "firewall" which was the SCL software bridge. Secondly, even though the use of SCL as middleware-enhanced modifications needed to plug CASPER onto EO-1, it was still not trivial to uplink and install CASPER without a significant amount of work. Figure 9 is the proposed next step whereby flight SW is loaded through the command link and begins to operate without rebooting the onboard computer as must be done with the SCL/CASPER method. The best analogy is the USB devices, which plug into desktop PC's, self configure and automatically begin to operate. We presently have a 


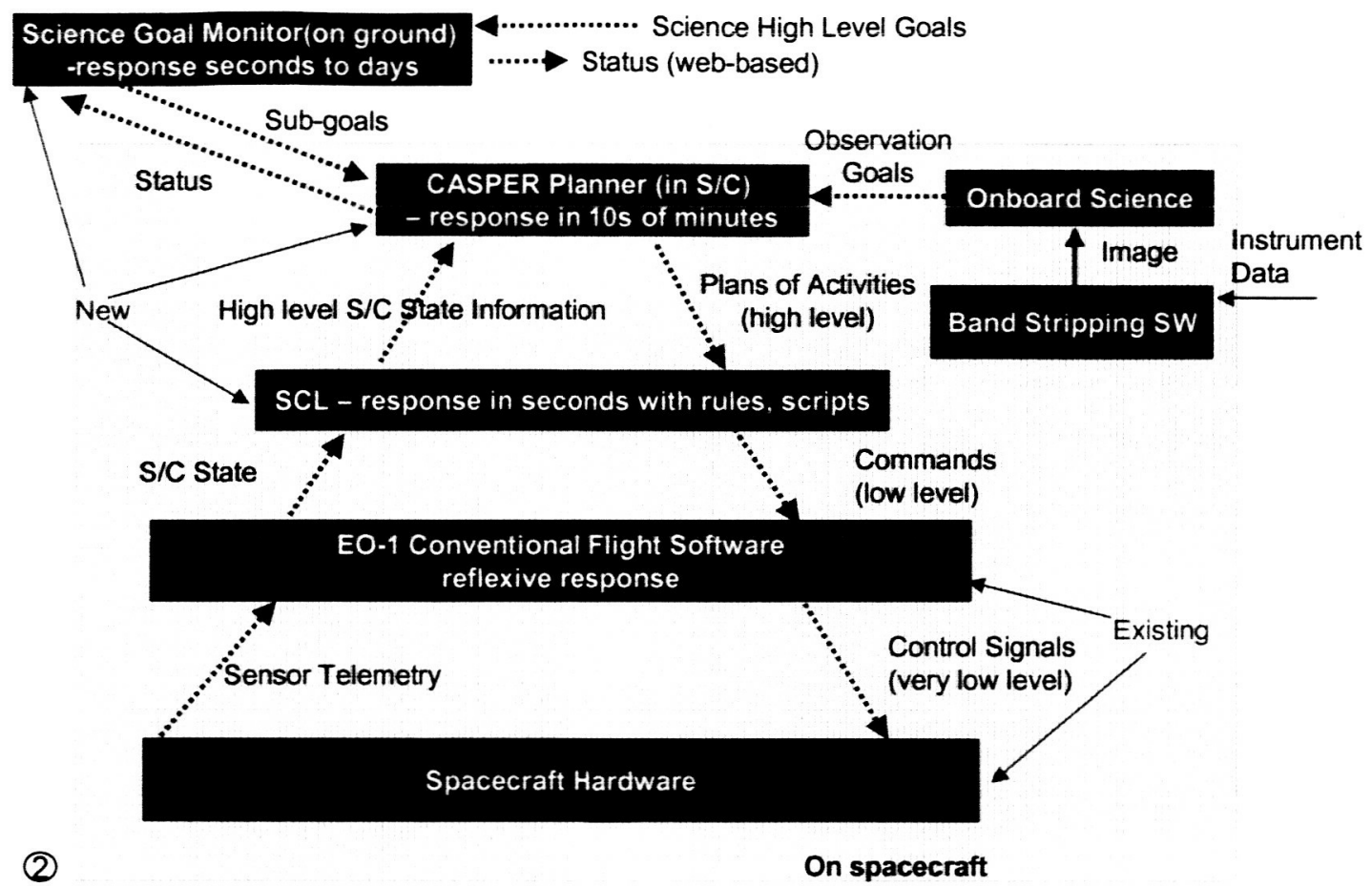

Figure 6 - Conceptual architecture for augmentation of EO-1 with both onboard Continuous Activity Scheduling Planning Execution and Replanning(CASPER) system and ground Science Goal Monitor (SGM) planners while EO-1 is on-orbit proposal that could demonstrate that capability on EO-1 if funding is located.

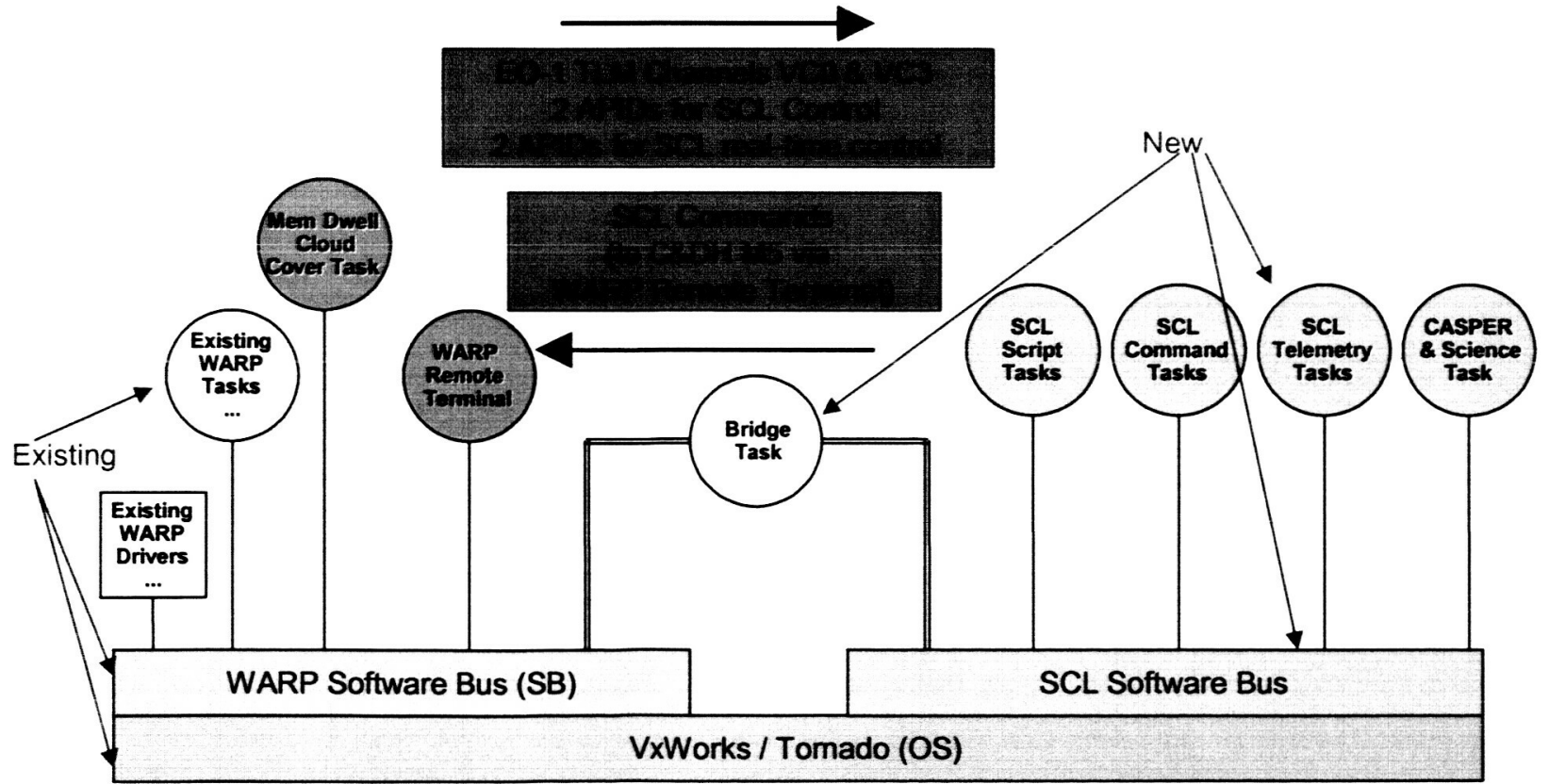

Figure 7 - Augmented software architecture using SCL as middleware to create an onboard dynamic software bus on the Wideband Advanced Recorder Processor (WARP) Mongoose, one of two Mongoose processors on EO-1. 


\section{Sensor Web Demonstration Scenario 1, National}

\section{Priority Wildfires, Performed 8-21-03}

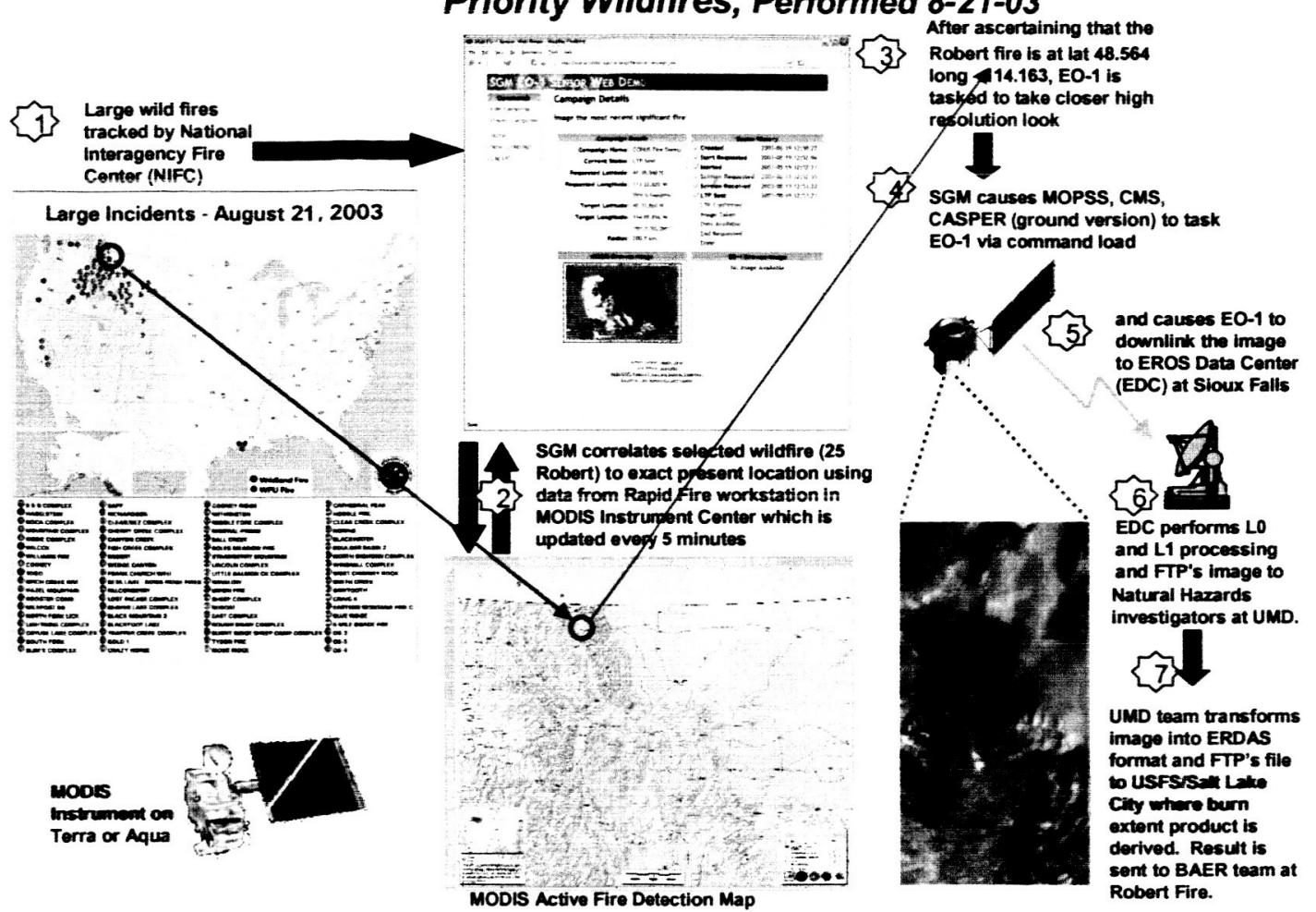

Figure 8 - EO-1 Sensor Web Fire Detection Operations Scenario Diagram from 8-21-03; Robert's Fire in Montana

\section{Developing a Sensor Web Framework Using EO-1 as the First Step}

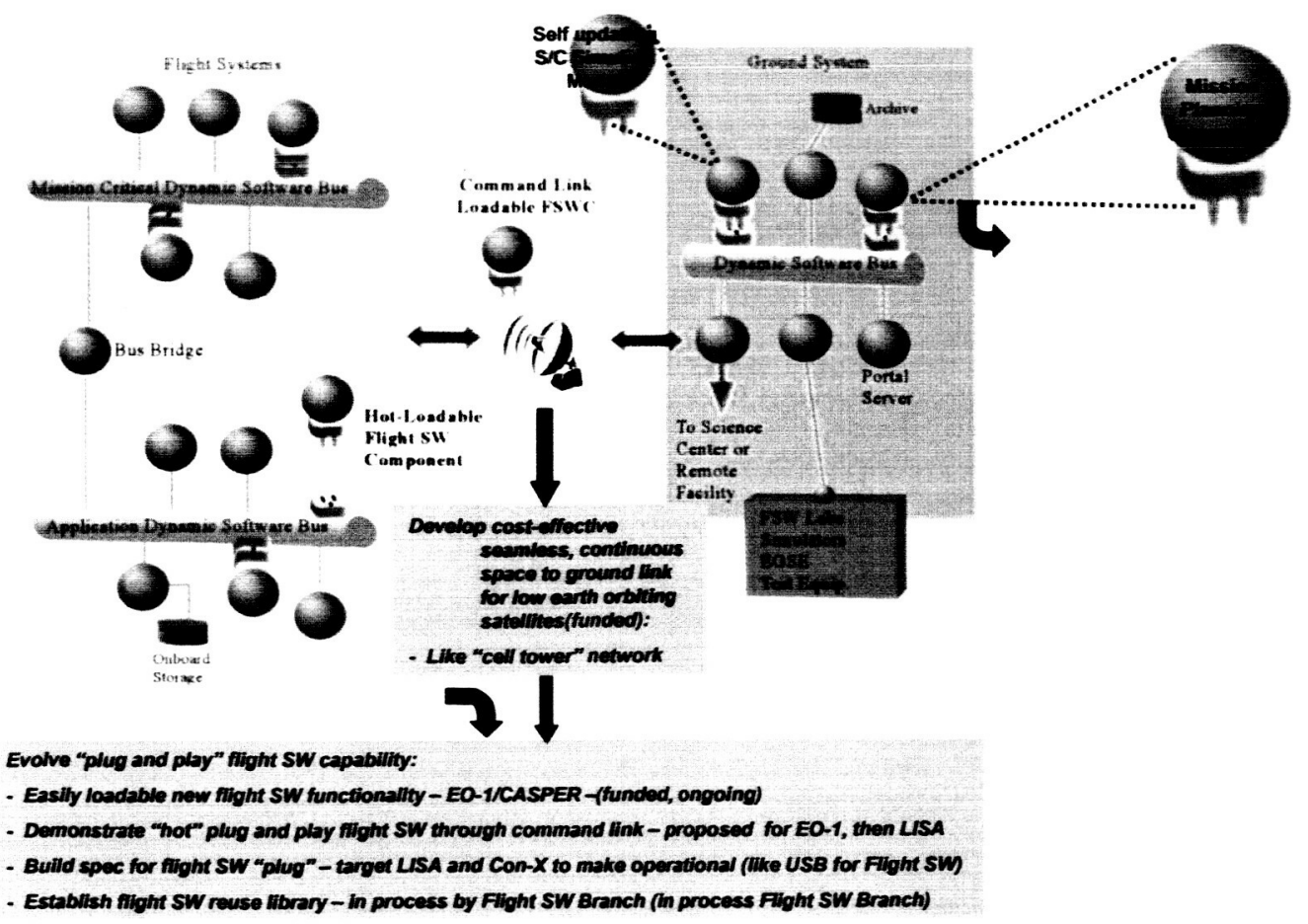

Figure 9 - Conceptual diagram to implement the higher level of plug and play for a sensor web. 


\section{Related SensorWeb Tasks Using EO-1 as a Testbed}

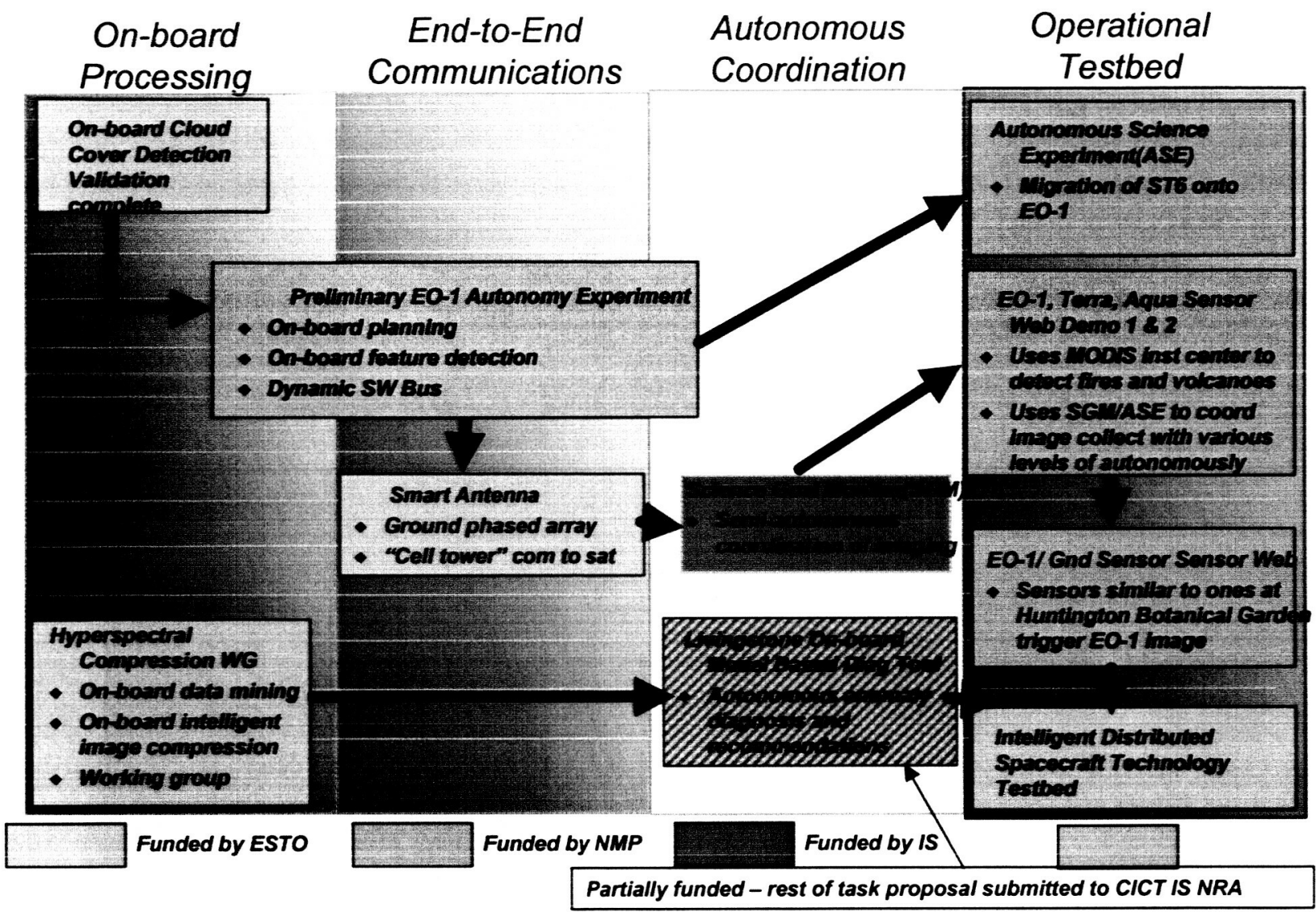

Figure 10 - Funded tasks on EO-1 Testbed.

\section{References}

\section{CONCLUSION}

The effort to construct a collaborative sensing system using heterogeneous satellites led to a different conclusion than might have been expected for future constellations.

Typically, one would expect that software reuse will be a big factor in making future constellations cost-effective. However, to get enough "economy of scales", it may not be enough to have reuse within one organization by

standardizing all of the engineering tools and internal reuse software libraries. Therefore standardizing on a messaging backplane, which allows interoperability may provide the capability to have "plug and play" components, which can leverage the effort of many organizations.
[1] Steve Chien, R. Sherwood, D. Tran, R. Castano, B. Cichy, A. Davies, G. Rabideau, N. Tang, M. Burl, D. Mandl, S. Frye, J. Hengemihle, J. Agostino, R. Bote, B. Trout, S. Shulman, S. Ungar, J. Van Gaasbeck, D. Boyer, M. Griffin, H. Burke, R. Greeley, T. Doggett, K. Williams, V. Baker, J. Dohm, "Autonomous Science on the EO-1 Mission," International Symposium on Artificial Intelligence, Robotics, and Automation in Space (i-SAIRAS 2003). Nara, Japan, May 2003.

[2] A. G. Davies, S. Chien, R. Wright, P. Cervelli, L. Flynn, V. Baker, R. Castano, B. Cichy, J. Dohm, T. Doggett, R. Greeley, K Williams, S. Frye, J. Jones, and the ASE and EO-1 Mission Teams (2003), "Streamlining Spacecraft Observation Response to Volcanic Activity Detection with a Ground and Space-based Sensor Web System." Abstract, AGU Fall Meeting 2003, San Francisco, CA.

[3] J.D. Dohm, S. Chien, G. Brakenridge, V. Baker, R. Castano, B. Caquard, B. Cichy, A. Davies, T. Doggett, R. Greeley, S. Nghiem, R. Sherwood, K. Williams, D. Mandl, S. Ungar, S. Frye, J. Jones, S. Grovsenor and the ASE and EO-1 Operations Teams (2003) "Ground and Space-based 
Sensor Web System: Streamlining Spacecraft Observation Response to Flood Detection," abstract, American Geophysics. Union Fall Meeting, San Francisco, 6-12 Dec 2003.

[4] Jeremy Jones, "Science Goal Driven Automation for NASA Missions: The Science Goal Monitor," AAAI Spring Symposium on Interaction Between Humans and Autonomous Systems Over Extended Operation, 2004.

\section{Author Bio}

Dan Mandl is presently the EO-1 Mission Director and the Ground Systems Manager for ST-5. Mr. Mandl led EO-1 through a flawless first year of technology validation operations and spearheaded an effort to convert EO-1 into an on-orbit testbed after the first year. Due to all of the streamlining in operations which included setting up a partnership with USGS to sell EO-1 imagery after the first year and exceeding expected results through the use of EO1 as a testbed, the EO-1 team was awarded the NASA Continuous Improvement Award. $\mathrm{He}$ is also the PI on a

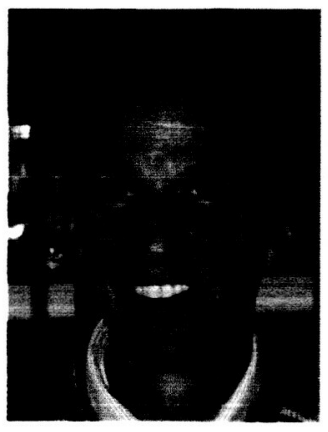
winning proposal to NASA Earth Science Technology Office(ESTO) to investigate techniques to create hybrid ground phased array antennas to lower the cost of antennas used to communicate to satellites and the PI on a recently completed investigation awarded by ESTO to demonstrate onboard cloud cover assessment using EO-1. Previously, he was the TRACE Ground System Development lead. His other jobs have included being the Small Explorer Control Center Systems Manager, a developer on other various missions such as COBE, GRO, UARS and EUVE. He has a BSEE from Univ. of Md and a Master's of Engineering Management from George Washington Univ.

\section{Acknowledgements}

The author would like to gratefully acknowledge the science support provided by Dr. Rob Sohlberg, Principal Investigator for the NASA Natural Hazards Program and Dr. Chris Justice, a fire investigator for the NASA Natural Hazards Program. Also, the author would like to gratefully acknowledge the support provided by Jacques Des Cloitures at the MODIS Rapid Response System for support provided in detecting and locating fires via the MODIS active fire map. Finally, the author would like to acknowledge the support provided by the EO-1 operations team and the EO-1 autonomy team which consisted of people from Goddard Space Flight Center and Jet Propulsion Laboratory. 